\title{
A Practical Implementation of an Agriculture Field Monitoring using Wireless Sensor Networks and IoT Enabled
}

\author{
Edmond Nurellari ${ }^{\dagger}$ and Saket Srivastava ${ }^{\dagger}$ \\ $\dagger$ School of Engineering, University of Lincoln, United Kingdom \\ Enurellari@lincoln.ac.uk, Ssrivastava@lincoln.ac.uk
}

\begin{abstract}
In this work, we consider the problem of designing a state of the art energy - ef ficient wireless sensor network (WSN) practically deployed in a large field. The sensor nodes (SNs) are tasked to monitor a large region of interest (ROI) and report their test statistics to the fusion center (FC) over a wireless fading channel. To maximize the lifetime of the WSN and enable long range communication with minimal transmit power, the long range wide area network (LoRaWAN) communication protocol is adopted. Each of the $\mathrm{SN}$ is designed and enabled with several state of the art sensors in order to estimate different and diverse parameters of interest (e.g., soil moisture, soil temperature, and salinity at different soil depth; barometric pressure, ambient humidity, leaf wetness, and etc.). The core feature of the proposed solution is that the SNs learn and adopt over the sensing time. This is very important in extending the operational lifetime of the WSN. The proposed system is validated through the infield experiments using few concept devices. Experimental results show that the proposed WSN features an effective large ROI monitoring with minimal number of SNs, a significantly reduced SN transmission power required and thus an extended WSN operational lifetime.
\end{abstract}

Index Terms-Wireless sensor network, IoT for agriculture, LoRaWAN, fading, energy efficiency.

\section{INTRODUCTION}

Defined as one of the most important emerging technologies, wireless sensor networks (WSNs), together with Internet of Things (IoT) [1] will revolutionize the world. In fact, the core component of the IoT paradigm is the WSN. Monitoring a region of interest (ROI) is one of the most important applications of WSNs [2]-[4]. We have witnessed their deployment in the last 10 years in several sectors such as health-care, building infrastructure, environment monitoring, agriculture and military [5]-[11]. Very recently, WSN have been designed to add intelligence to the agriculture sector and hence enable smart IoT based farming [12]. In such application, monitoring different environmental parameters such as temperature, humidity, weather station data, leaf wetness, soil temperature/humidity and many other parameters are of particular interest. The monitoring of these parameters not only allows time and cost minimization but also enables agriculture productivity to be maximized. For example, since the mid1990s, increases in UK wheat yields on-farm have stalled, while oil-seed rape farm yields have fluctuated wildly since the

This work was supported in part by the Biotechnology and Biological Sciences Research Council through the Industrial Strategy Challenge Fund. 1980s with yields now little different to those of thirty years ago. This is despite genetic yield gain continuing to deliver progress at more than $0.5 \%$ per year for winter wheat and $2 \%$ for oil-seed rape [13]. Management intensity is considered one avenue to pursue to overcome existing limitations.

Generally, the local sensing process is orientated towards estimating some different parameters of interest which can then be optimally combined to arrive at an informed decision. This decision can then be relayed in a pre-specified manner or can be employed for on-field actuation (e.g., in the context of agriculture this can be irrigation switches control, crop harvesting decision time, actuation of self-autonomous vehicle operating in-field, and etc.). We note that the unattended, reliable and continued operation of a WSN over many years is often desirable. Furthermore, designing intelligent WSNs that efficiently utilize the on-board limited energy resources and cope with the impairments in a wireless communication channel is very important.

While there are some theoretical studies and considerations of WSNs applied to farming [14]-[16] to name but just a few, there is still limited practical work done towards designing and enabling WSNs for agriculture purposes. Reference [17] deals with efficiency of feedback control method in greenhouse crop irrigation and advocates the water saving by this means. Authors in [18] consider developing SNs that can measure parameters which affect crop growth and quality and design a visualization platform to output the data. Some preliminary initiatives towards developing practical IoT enabled agriculture solution can be found in [19].

Now, the existing work either deal with a single SN deployment with limited number of equipped and low resolution sensors or provide some basic data collection algorithms without any insight into the problem. Furthermore, none of the previous work deal with energy - efficiency and the ROI coverage maximization constraint to a fixed given number of SNs.

This work contributes in designing a private energy-efficient WSN to be practically deployed in an agriculture field to monitor a large ROI (see Fig. 2). Being geographically dispersed to cover large areas, the SNs are constrained in both bandwidth and power. We design state of the art LoRaWAN enabled SNs that feature low power consumption and long range communication equipped with several state of the art 
agriculture sensors (see details later in Section III-B). We propose a learn and adapt algorithm to extend the operational lifetime of the WSN and to deal with the imbalanced load among the SNs. The communication links (SNs-to-FC) are tested for communication channels experiencing shadowing and multipath fading. We show that the proposed system effectively deals with the energy imbalance in a WSN.

\section{Design Challenges IN WSNS}

While there are several design issues and challenges in WSNs, here we briefly discuss the three most important issues that are related to bandwidth/energy-constrained WSNs deployed across the agriculture sector.

1) Low Power Hardware: Clearly, the biggest design constraint in WSNs still remains the power consumption. Even-though the SNs are being designed using lowpower micro controllers, their power dissipation is still orders of magnitude too high. For a survey on hardware systems for WSNs, we refer the reader to [25] and see references therein.

2) Resource Constraints: Because the SNs are battery operated devices with limited on-board energy, both the system lifetime and communication bandwidth (BW) are restricted. While designing the algorithms to be used by the SNs, both the signal processing and communication should be carefully designed to consume minimal energy in order to extend the lifetime and improve the overall reliability of the WSN. In this work, we consider several distributed estimation parameters with SNs intelligently reporting their local observations to the $\mathrm{FC}$ when it is necessary to do so.

3) Network Security: Being geographically dispersed to cover large areas, the $\mathrm{SNs}$ are usually unattended and this makes them vulnerable to different types of attacks or hardware malfunctioning. The overall sensing and estimation performance strongly depends on the reliability of these SNs in the network. While fusing the data received by the spatially deployed SNs allows the FC to make a reliable decision regarding the soil moisture mapping, it is possible that one or more SNs malfunctioning (compromised by an attacker) are sensing noisy (deliberately falsify their) local observations to degrade the overall FC detection performance. While there are many types of security threats, these are out of this work scope. For further details on network security we refer the reader to [22] and references therein.

For other design issues such as SN localization, medium access control (MAC) protocols, time synchronization, hardware design and routing protocols in an energy constrained WSN, we refer the reader to [6].

\section{SYSTEM MODEL}

Consider the problem of monitoring a large agriculture ROI by a WSN consisting of a gateway, a FC, and $M$ spatially distributed SNs all equipped with a single antenna and report to the FC via a dedicated point-to-point communication link. The

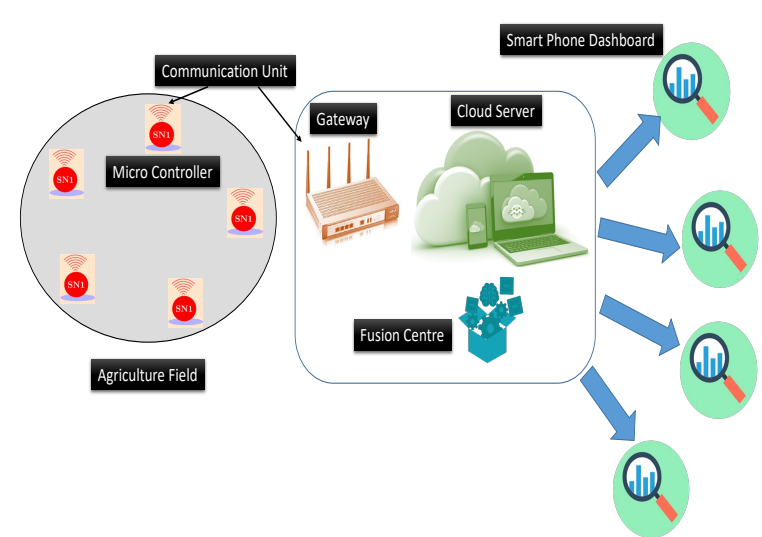

Fig. 1. System components and hierarchical organization of information flow from peripheral sensor nodes to fusion center, and finally to the end user.

wireless channels SNs-to-FC are set to experience shadowing as well as small scale fading ${ }^{1}$. The case where the spatially distributed SNs report to the FC via a dedicated parallel access channel (PAC) is investigated in e.g., [3], [20].

Next, we discuss the local deployed SN and its features.

\section{A. Sensor Node}

In this work, we have considered deploying 9 homogeneous SNs across a 9 hectares field. Each of the $\mathrm{SN}$ is equipped with $6600 \mathrm{mAh}$ rechargeable battery and designed to have solar energy harvesting capabilities such that in-field post innervation is none (or minimal). The encapsulated box is robust waterproof IP65 enclosure and hence can be seamlessly deployed in any harsh environment. The SNs feature an internal secure digital (SD) card, FAT32 file system is used that can store a capacity of approximately 70 million measurements. To extend the operational lifetime of the SN and enable long range communication such that a large ROI is efficiently monitored, the LoRaWAN communication protocol is adopted. This technology effectiveness will be practically tested against the communication channels (i.e., SN-to-FC) experiencing multipath fading and shadowing due to close by forest and big animals in-field. In the experimental result, for the chosen scenario, we will experiment and show the minimum receiver sensitivity (the maximum distance) such that an effective communication can be still maintained. A core feature of the $\mathrm{SN}$ is that it can service up to 8 different low-power sensors simultaneously (e.g., ambient temperature sensor, soil moisture sensor, leaf wetness sensor, and etc.). This enables cost-effective monitoring of a large set of local parameters using a single master unit and hence its suitability in agriculture monitoring where cheap and reliable solutions are vital in this sector.

Next, we discuss the sensing process and sensors integrated in this proposed solution.

\footnotetext{
${ }^{1}$ The small scale fading is assumed to be time-variant over a coherence time $\tau$.
} 

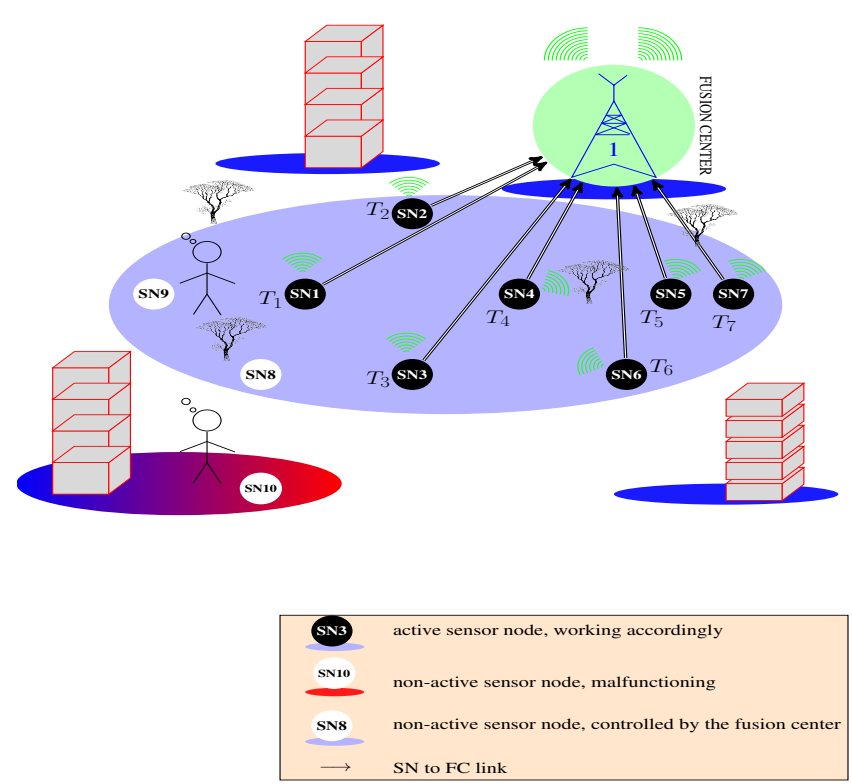

Fig. 2. Schematic communication architecture among peripheral active SNs and FC. The $i$ th active $\mathrm{SN}$ generates a test statistic $\left(T_{i}\right)$ and communicates this to the FC directly. There are two categories of non-active SNs: a) non active due to malfunctioning; b) non-active due to FC control.

\section{B. Local Sensing and Sensors}

For this initial feasibility study and proof of concept, we have chosen a grass field that from our preliminary analysis is estimated to have some soil variations across the field. We will be deploying uniformly 9 state of the art (both from the agriculture and electronic perspective) soil moisture probes across this 9 hectares field to try and establish an accurate soil moisture map at different depths. This capacitive soil moisture probe [21] (see Fig. 3), enabling near continuous measurements, is $30 \mathrm{~cm}$ in length and with sensors (soil moisture, soil temperature, and salinity) placed at $10 \mathrm{~cm}$ apart. Based on the initial results obtained, we then would be able to re-deploy the units in an informed manner in an attempt to further improve the soil moisture mapping.

Now, we would like to make it clear that while the above soil measurement probe shown in Fig. 3 yields superior results from agriculture perspective, it was not IoT enabled (i.e., did not integrate with the state of the art low power SNs and microcontrollers (e.g., [24])). In this work, we have integrated this soil moisture probe with the low power hardware [24] such that a state of the art IoT solution is obtained. Finally, some of the probe's parameters can be found in [21] and are also depicted here in Table I.

Finally, to enable a minimal post-innervation in practical WSNs and increase the operational life time of the WSN, the energy resources have to be constantly monitored. Each local SN monitors its battery level, sense and stores the solar energy harvested such that an energy budget profile can be built. Using both the current battery level and this budget profile (built overtime), each SN decides on how frequently to sample the

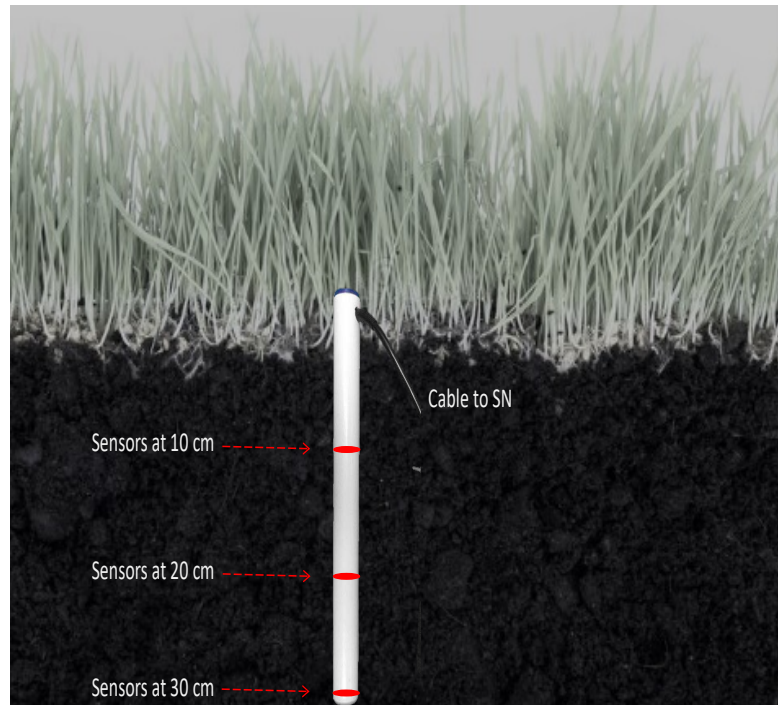

Fig. 3. Actual probe deployment inside the ground for in-field soil monitoring.

TABLE I

Drill \& Drop Soil Measurement Probe Specifications.

\begin{tabular}{|c|c|c|c|}
\hline Types of Sensors (at each depth) & Moisture & Temperature & Salinity \\
\hline Resolution & $1: 10000$ & $1: 6000$ & $0.3^{\circ} \mathrm{C}$ \\
\hline Moisture Precision & $\pm 0.03 \%$ vol. & $\mathrm{x}$ & $\mathrm{x}$ \\
\hline Temperature Accuracy & $\mathrm{x}$ & $\pm 2{ }^{\circ} \mathrm{C} @ 25^{\circ} \mathrm{C}$ & $\mathrm{X}$ \\
\hline Operating Temperature & $-20^{\circ} \mathrm{C}-60^{\circ} \mathrm{C}$ & $-20^{\circ} \mathrm{C}-60^{\circ} \mathrm{C}$ & $-20^{\circ} \mathrm{C}-60^{\circ} \mathrm{C}$ \\
\hline
\end{tabular}

field (i.e., local sampling rate). This adaptive rating enables an effective way of resources management and an increase of the WSN operational lifetime. We note again that a continuous and reliable operational WSN is highly desirable in practice.

The SNs, depending on how are deployed and used, can vary from being extremely tiny devices [23] to relatively large embedded platforms [24]. In general, a SN consists of limited signal processing capabilities, sensing device(s), a transceiver, limited memory capacity, and on-board power [6]. These devices have wireless communications capability that makes them suitable in a variety of applications as described above. However, there are a numerous challenging problems in designing WSNs that we describe next in Section II.

Next, we discuss the communication architecture and data collection scheme.

\section{Communication Architecture and Data Collection}

There are different WSNs architectures depending on how the SNs take decision and exchange information with other SNs in the network or with the fusion center (FC) [22] (see Fig. 2). We briefly mention here three of the different architectures and focus in more details to the one that we will be using in this work; 1) The Centralized Architecture (we consider this here), where there are mainly spatially distributed SNs that report to the FC. There is no inter-sensors collaboration. 2) The Distributed Architecture, where there 
is no FC and the SNs collaborate with each other in achieving the common goal. 3) The Hybrid Architecture, where there is a FC and there is also inter-sensor nodes collaboration.

For other design issues such as resource allocations, fusion rules, and network security in an energy constrained WSN, we refer the reader to [22].

In this work, as stated above, we adopt the Centralized Architecture where each of the local SN report its data to the FC where further processing is performed. Every SN in the network decides in transmitting the local data to the FC by performing a two - step decision algorithm:

(i) FIRST STEP: Every SN in the network compares the current data with the previous sensed data. For e.g., consider the current temperature sensor data obtained at an instantaneous discrete time $n=\frac{t}{\Delta_{\mathrm{s}}}$, at each $\mathrm{SN}\left(s_{i}(n)\right)$, is further corrupted by $\operatorname{AWGN}\left(w_{i}(n) \sim \mathcal{N}\left(0, \sigma_{i}{ }^{2}\right)\right)$ :

$$
y_{i}(n)=s_{i}(n)+w_{i}(n)
$$

Similarly, at discrete time $n+1$, the temperature at the $i^{t h} \mathrm{SN}$ will be:

$$
y_{i}(n+1)=s_{i}(n+1)+w_{i}(n+1)
$$

Now, the $i^{\text {th }}$ SN performs the decision at discrete time $n$ based on the following condition:

$$
\left.\begin{array}{l}
\text { if }\left|y_{i}(n)-y_{i}(n+1)\right| \geq \Lambda_{f}, \text { go to SECOND STEP } \\
\text { if }\left|y_{i}(n)-y_{i}(n+1)\right|<\Lambda_{f} \text {, do not transmit to FC }
\end{array}\right\}
$$

where $\Lambda_{f}$ is the decision threshold and it is a design parameter in general.

(ii) SECOND STEP: All the SNs with positive transmission decision in (3) and step 1 perform now the local decision on the battery level at discrete time $n$ as follows:

$$
\left.\begin{array}{l}
\text { if } \beta_{j}(n) \geq \tau_{f}, \text { transmit to } \mathrm{FC} \\
\text { if } \beta_{j}(n)<\tau_{f}, \text { go to deep sleep }
\end{array}\right\}
$$

where $j$ is the first step positive transmission $\mathrm{SN}$ index (i.e., of those SNs that with decision rule in (3) are set to transmit to FC), $\beta_{j}(n)$ represents the $j^{\text {th }}$ SN's battery level at discrete time $n$, and $\tau_{f}$ is the battery level decision threshold (also a design parameter).

Clearly, from the above, a SN is set to transmit to the FC if both $\left|y_{i}(n)-y_{i}(n+1)\right| \geq \Lambda_{f}$ and $\beta_{j}(n) \geq \tau_{f}$ are satisfied. Otherwise, the SN is set to stay in sleeping mode for a defined period of time.

Now, the energy - efficient algorithm (with two - step decision rule (3) and (4) can be summarized in Algorithm1.

\section{EXPERIMENTAL RESULTS}

We evaluate experimentally the performance of our proposed energy - efficient low power WSN solution. We consider a WSN deployed in a $300 \mathrm{~m} \times 300 \mathrm{~m}$ ROI (i.e., the grass field) with $M=9$ SNs and square type SN geometry. The FC is located at a distance of $950 \mathrm{~m}$ non line of sight

\section{Algorithm1: Energy Efficient Two-Step Transmission \\ Decision Algorithm \\ STEP 1: Choose $\Lambda_{f}$ and evaluate (3), $\forall i$; \\ if $\left|y_{i}(n)-y_{i}(n+1)\right| \geq \Lambda_{f}$, go to STEP 2 \\ if $\left|y_{i}(n)-y_{i}(n+1)\right|<\Lambda_{f}$, go to STEP 3}

STEP 2: Choose $\tau_{f}$ and perform the decision (4), $\forall j$, to estimate the transmission mode on/off;

if $\beta_{j}(n) \geq \tau_{f}$, go to STEP 4

if $\beta_{j}(n)<\tau_{f}$, go to STEP 3

STEP 3: Go to a deep sleep mode for 30 minutes and then go to STEP 1;

STEP 4: Transmit all the data packets to the FC, then go to STEP 3;

(NLOS) from the center of the ROI and the distances between the SNs are chosen large enough such that the machines can service the field as normally without any intervention (e.g., the grass cutter machine). This spatial configuration is shown in Fig. 4. Because the grass field is frequently visited by big animals and cattle, each of the SN unit is protected by a physical fencing system with an area of $1 \mathrm{~m}^{2}$.

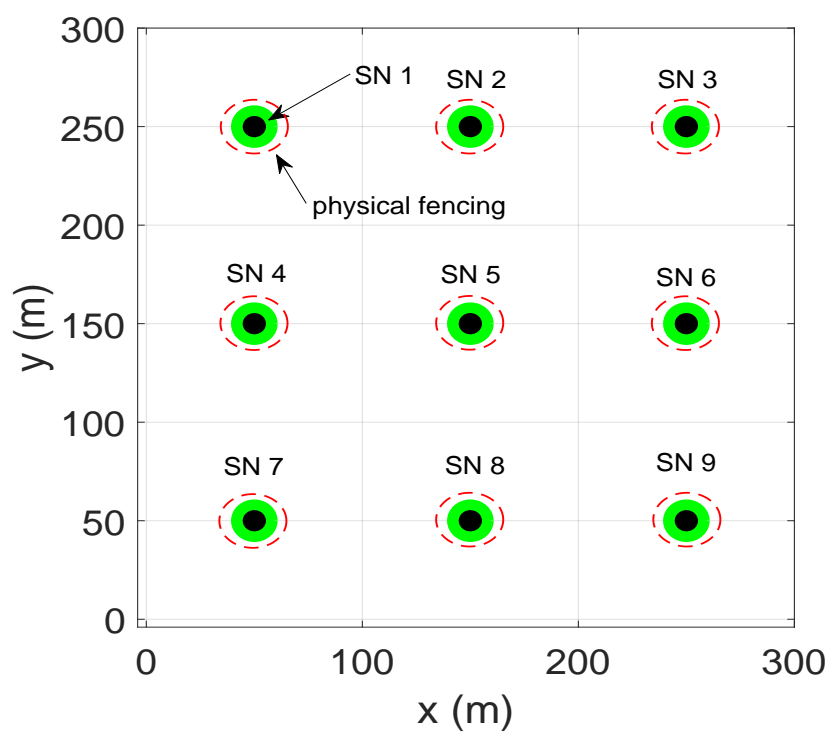

Fig. 4. Spatial configuration of the WSN where the SNs are represented with green and the red dashed circle represent the physical fencing system.

In Fig. 5, we show the data rate for SN 2 against the received signal strength indication (RSSI) recorded for a period of time from $23^{\text {rd }}$ of April until $2^{\text {nd }}$ of July. As expected, SN data rate increases with improving RSSI. This adaptive data rate transmission is very important in not only receiving the data packets without error but also saving the SN's energy. For example, for the cases where the communication channel is experiencing deep fading and shadowing, the SN does not transmit any data to the FC in order to conserve energy.

Now, to further validate our system set up and the SN's in-field spatial deployment, in Fig. 6 we observe the RSSI 


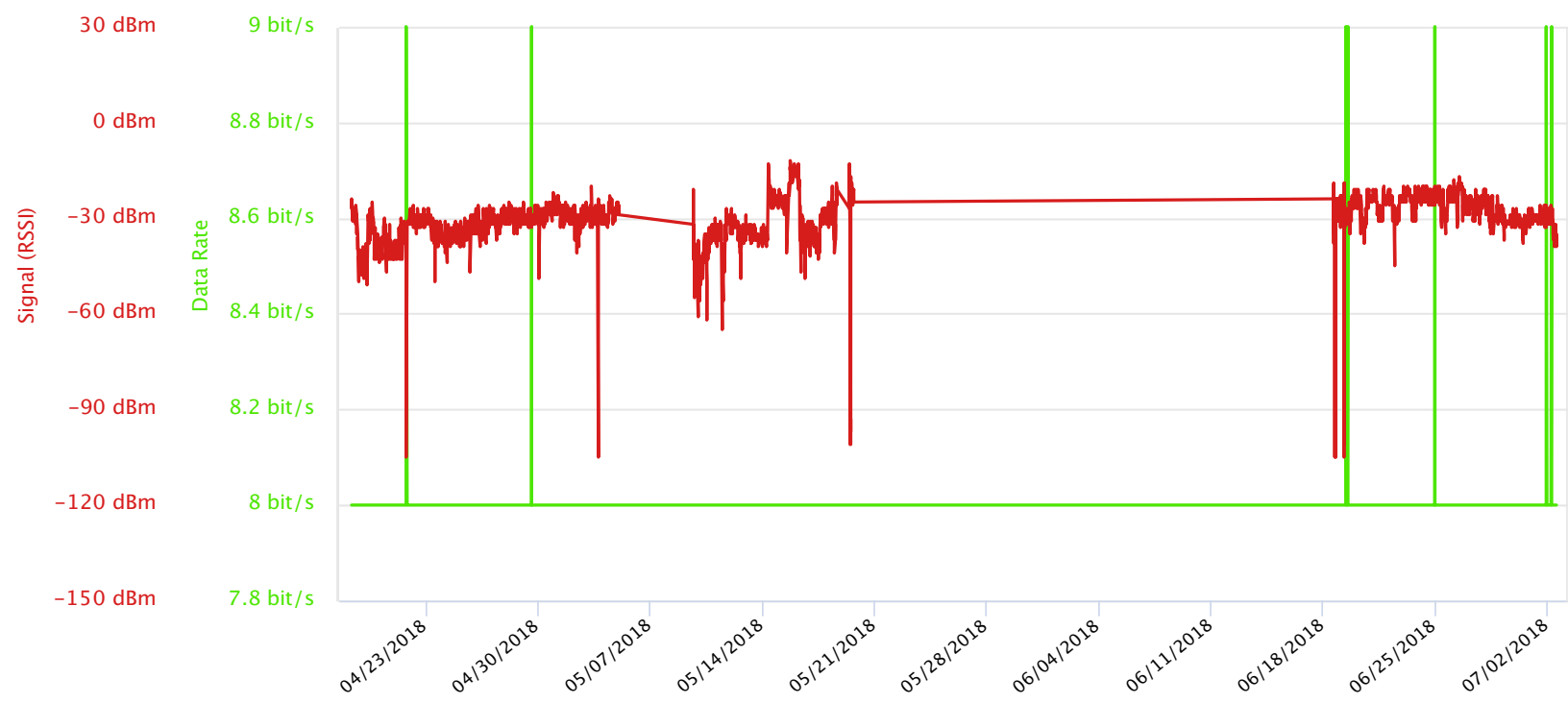

Fig. 5. The data rate for SN 2 against the received signal strength indication (RSSI) plotted for a period of time from 23/04/2018 up to 02/07/2018.

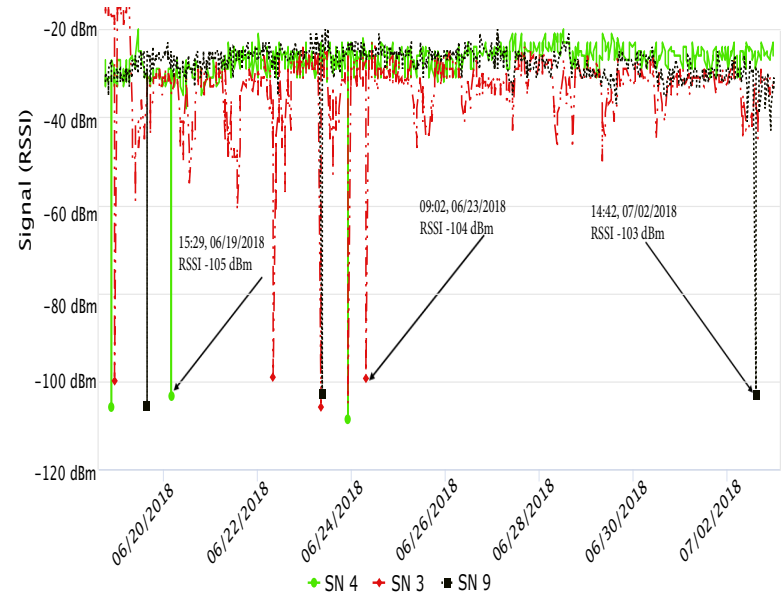

Fig. 6. Received signal strength indication (RSSI) profiles for 3 arbitrarily chosen SNs (specifically SN 3, SN 4, and SN 9).

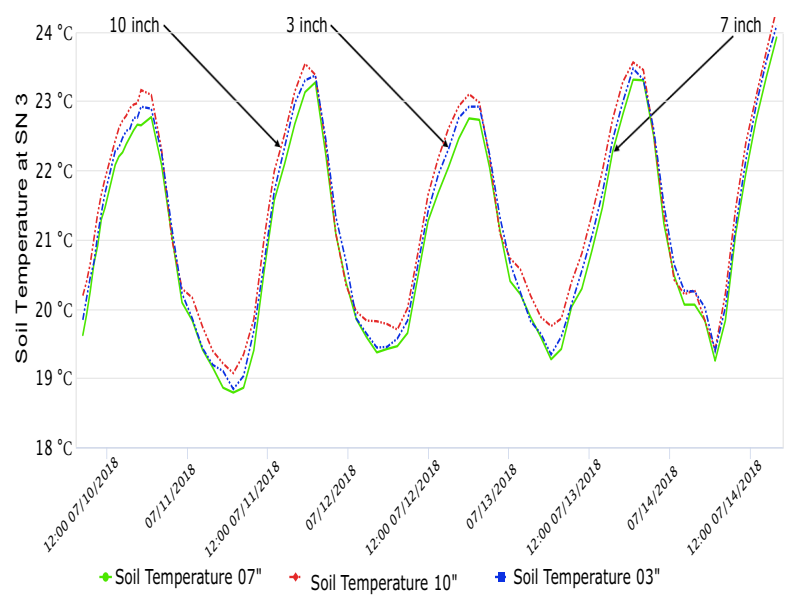

Fig. 7. Soil temperature profile at different depths for SN 3, plotted for a period of time from 10/07/2018 up to 15/07/2018. for three arbitrary chosen SNs. Clearly, all the SNs are experiencing deep fading and/or path loss at some particular time instant. For example, SN 9 is experiencing the worst communication channel on $19^{\text {th }}$ of June at $15: 29$, on $23^{\text {rd }}$ of June at 09:02, and on $2^{\text {nd }}$ of July at 14:42. However, most of the time, the signal strength is high such that a reliable communication with the FC is maintained. Similar observation can be seen for SN 3 and SN 4. Few spikes are observe at some time instant indicating challenging channel conditions. This is as expected since the communication path between the SNs and the FC is affected by the high trees and big animals in the field. However, note that the receiver sensitivity adapted in this system is down to $-136 \mathrm{dBm}$.

In Fig. 7, for an arbitrary chosen SN (i.e., SN 3), we plot the soil temperature at three different depths. As expected, the biggest variation is observed among the soil temperature measured at 3 inch and 10 inch.

Finally, to demonstrate the reliability of the proposed system on the soil moisture monitoring and mapping, in Fig. 8, for some arbitrary chosen SNs (i.e., SN 4, SN 7, SN 8, and SN 9), we plot the soil moisture profiles at 3 inch depth sampled across the field. Clearly, a variation between the selected SNs' measurements is observed. For example, the biggest variation is observed for the SNs far apart from each other (e.g., SN 4 and SN 9). This is as expected since the soil content at these locations will be much more different.

\section{CONCLUSIONS}

In this paper, we propose an energy - efficient WSN for soil moisture monitoring and mapping. To further extend the WSN operational lifetime, we have proposed a simple but efficient algorithm such that the SN transmit power can be significantly reduced. Finally, we have also shown that the proposed WSN results in a reliable and operational system even in the worst fading communication channel case. Future 


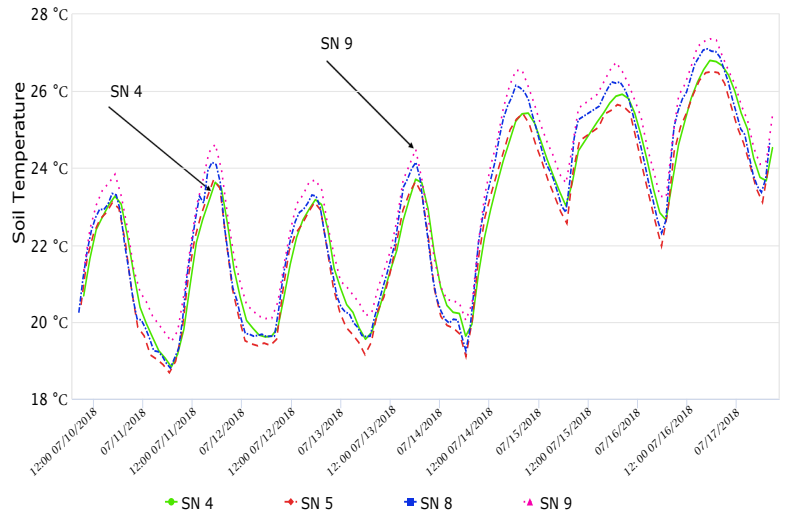

Fig. 8. Soil temperature profile at 3 inch depths for SN 4, SN 5, SN 8, and SN 9 plotted for a period of time from 10/07/2018 up to 17/07/2018.

work will further investigate the analysis of the soil moisture mapping by considering the data at 3 different depths such as soil temperature, salinity, and soil moisture. Future work will also investigate the minimum SNs number required such that the obtained results are within the acceptable accuracy range (a design parameter). This analysis is important from practical perspective as by reducing the SNs number so does the overall cost of WSN.

\section{REFERENCES}

[1] G. Kortuem, F. Kawsar, V. Sundramoorthy, and D. Fitton, "Smart objects as building blocks for the Internet of things," in IEEE Internet Computing, vol. 14, no. 1, pp. 44-51, Feb. 2010.

[2] C-Y Chong, SP Kumar, "Sensor networks: evolution, opportunities, and challenges," in Proc. IEEE., vol. 91, no.8, pp. 12471256, Aug. 2003.

[3] S. Aldalahmeh, M. Ghogho, D. McLernon, and E. Nurellari, "Optimal fusion rule for distributed detection in clustered wireless sensor networks", EURASIP Journal on Advances in Signal Process., 2016:5, Jan. 2016.

[4] I. Akyildiz, W. Su, Y. Sankarasubramanian, E. Cayirci, "A survey on sensor networks", in IEEE Communications Magazine, vol. 40, no.8, pp. 102-114, Nov. 2002.

[5] E. Nurellari, D. McLernon, and M. Ghogho, "Distributed TwoStep Quantized Fusion Rules via Consensus Algorithm for Distributed Detection in Wireless Sensor Networks," in IEEE Transactions on Signal and Information Processing over Networks, vol. 2, no. 3, pp. 321-335, Sept. 2016.

[6] B. Sadler, "Fundamentals of energy-constrained sensor network systems," in IEEE Aerospace and Electronic Systems Magazine, vol. 20, no. 8, pp. 17-35, Aug. 2005.

[7] E. Nurellari, D. McLernon, and M. Ghogho, "A Secure Optimum Distributed Detection Scheme in Under-Attack Wireless Sensor Networks," in IEEE Transactions on Signal and Information Processing over Networks, vol. 4, no. 2 , pp. 325-337, Jun. 2018.

[8] I. F. Akyildiz, W. Su, Y. Sankarasubramaniam, and E. Cayirci, "A survey on sensor networks," in IEEE Communication Magazine, pp. 102-114, Aug. 2002.

[9] E. Nurellari, D. McLernon, M. Ghogho, and S. Aldalahmeh, "Distributed Binary Event Detection Under Data-Falsification and Energy-Bandwidth Limitation," in IEEE Sensors Journal, vol. 16, no. 16 , pp. 6298-6309, Aug. 2016.

[10] G. J. Pottie and W. J. Kaiser, "Wireless integrated network sensors," in Communications of the ACM, vol. 43, no. 5, pp. 51-58, May 2000.
[11] E. Nurellari, S. Aldalahmeh, M. Ghogho, and D. McLernon, "Quantized Fusion Rules for Energy-Based Distributed Detection in Wireless Sensor Networks," in Proc. Sensor Signal Processing for Defence (SSPD), Edinburgh, Scotland, pp. 1-5, 8-9 Sept. 2014.

[12] A. Tzounis, N. Katsoulas, T. Bartzanas, C. Kittas, "Internet of Things in agriculture, recent advances and future challenges", in Biosystems Engineering, pp. 31-48, 164(2017).

[13] AHDB and HGCA, "Provisional AHDB/HGCA $2012 \mathrm{Ce}-$ real Quality Survey Estimates", pp. 1-4, 31 Aug. 2012 [Online] Available at: https://cereals.ahdb.org.uk/media/5338/ CQS_First_Provisional_Estimates12.pdf, Accessed: Jun. 2018.

[14] O. Pandithurai, S. Aishwarya, B. Aparna and K. Kavitha, "Agrotech: A digital model for monitoring soil and crops using internet of things (IOT)," in 3rd Int. Conf. on Science Tech. Engineering \& Management (ICONSTEM), Chennai, pp. 342-346, 2017.

[15] A. Kumar, A. Surendra, H. Mohan, K. M. Valliappan and N. Kirthika, "Internet of things based smart irrigation using regression algorithm," in Int. Conf. on Intelligent Computing, Instrumentation and Control Technologies (ICICICT), Kannur, pp. 1652-1657, 2017.

[16] A. J. Rau, J. Sankar, A. R. Mohan, D. Das Krishna and J. Mathew, "IoT based smart irrigation system and nutrient detection with disease analysis," in IEEE Region 10 Symposium (TENSYMP), Cochin, pp. 1-4, 2017.

[17] I. Mat, M. R. Mohd Kassim, A. N. Harun and I. Mat Yusoff, "IoT in Precision Agriculture applications using Wireless Moisture Sensor Network," in IEEE Conference on Open Systems (ICOS), Langkawi, pp. 24-29, 2016.

[18] K. O. Flores, I. M. Butaslac, J. E. M. Gonzales, S. M. G. Dumlao and R. S. J. Reyes, "Precision agriculture monitoring system using wireless sensor network and Raspberry Pi local server," in IEEE Region 10 Conference (TENCON), Singapore, pp. 3018-3021, 2016.

[19] R. K. Jha, S. Kumar, K. Joshi and R. Pandey, "Field monitoring using IoT in agriculture," in Int. Conf. on Intelligent Computing, Instrumentation and Control Technologies (ICICICT), Kannur, pp. 1417-1420, 2017.

[20] J.T. Meng, J.R. Yuan, S.-Z. Feng, Y.J Wei, “An Energy Efficient Clustering Scheme for Data Aggregation in Wireless Sensor Networks," in Journal of Computer Science and Technology, vol. 28, no. 3, pp. 564-573, May 2013.

[21] The Sentek technologies, "Sentek Drill \& Drop Encapsulated Soil Moisture, Salinity and Temperature Probe", Available at: http://www.sentek.com.au/downloads/downloads.asp? TypeID=7\&FolderID=16 Accessed: 25 Jun. 2018.

[22] E. Nurellari, "Distributed Detection and Estimation in Wireless Sensor Networks: Resource Allocation, Fusion Rules, and Network Security", pp. 1-206, University of Leeds, Feb. 2017.

[23] B. Warneke, M. Last, B. Liebowitz, and K. S. J. Pister, "Smart dust: communicating with a cubic millimeter computer," Computer, vol. 34, no. 1, pp. 44-51, Jan. 2001.

[24] The Libelium company, "Wireless Sensor Networks with Waspmote and Meshlium", Available at: http: //www.libelium.com/v11-files/documentation/mesh_extreme/ wsn-waspmote_and_meshlium_eng.pdf Accessed: Feb. 2014.

[25] M. Hempstead, M. J. Lyons, D. Brooks, and G-Y Wei, "Survey of Hardware Systems for Wireless Sensor Networks," Journal of Low Power Electronics, vol. 4, pp. 1-10, 2008.

[26] S. Barbarossa, S. Sardellitti, and P. Di Lorenzo, "Distributed Detection and Estimation in Wireless Sensor Networks," In Rama Chellappa and Sergios Theodoridis eds., Academic Press Library in Signal Processing, vol. 2, Communications and Radar Signal Processing, pp. 329-408, 2014. 